\title{
Distance learning of psychology students in the context of a pandemic: an analysis of the experience gained
}

\author{
Zoya G. Khanova ${ }^{1}$, Muslimat $G$. Akhmedova $^{2 *}$, and Olga I. Kaiasheva ${ }^{3}$ \\ ${ }^{1}$ Moscow University for Industry and Finance "Synergy", Department of Psychology, Moscow, \\ Russia \\ ${ }^{2}$ Financial University under the Government of the Russian Federation, Department of Humanities, \\ Moscow, Russia \\ ${ }^{3}$ Moscow Region State University, Department of Counseling Psychology, Mytishchi, Russia
}

\begin{abstract}
The conditions of the COVID-19 pandemic have led to difficulties in teaching students majoring in psychology, and the need to develop recommendations for universities to improve the system of distance learning at higher education institutions. Training of practical psychologists requires mandatory full-time participation in sessions of psychological counseling, passing supervision, familiarization with the leading areas of psychological counseling and psychotherapy, psychodiagnostics, and psychocorrective measures. These aspects are complicated by the restrictions caused by the pandemic, which complicates also the subsequent internship based on psychological centers, and the need to conduct practical training courses in a remote format. The purpose of the present research is to identify opportunities to improve the efficiency of distance learning for students majoring in psychology as well as university teachers in the context of the pandemic. The developed recommendations will be useful for improving the learning process of students of psychological specialties at higher education institutions. The proposed recommendations concern changes in the schedule of the university curriculum, and providing qualified support to teachers and students from the university's psychological service and administration, implementing master classes for university practitioners, providing free access to video recordings of sessions of leading psychology consultants, as well as free practical online conferences and webinars of professional psychological and psychotherapeutic communities.
\end{abstract}

Keywords: higher education, online learning, reflection, COVID-19.

\section{Introduction}

Studies of the problem of distance learning in the context of the pandemic are focused on identifying its main advantages and disadvantages, the possibilities of integration with traditional learning to improve the quality of students' education. The teachers' objective in

\footnotetext{
*Corresponding author: muslima11@rambler.ru
} 
the current circumstances is to promote the physical and psychological safety of students in the context of the COVID-19 pandemic [1].

The purpose of the present research is to identify opportunities to improve the efficiency of distance learning for psychology students and university teachers in the context of the pandemic.

Objectives of the research are as follows: to conduct theoretical analysis and generalization of scientific literature on the studied problem; to identify the shortcomings and positive aspects of distance learning in the course of training psychology students in the context of the pandemic; to offer recommendations for improving the level of training of students in distance learning in the context of the pandemic.

Hypothesis of the research is as follows: the analysis of the requirements for professional activities, and the development of recommendations based on the results of the study will contribute to improving the efficiency of distance learning of psychology students.

Justification of novelty: the authors propose recommendations for improving the level of training of psychology students in distance learning in the context of the pandemic.

Crisis-ridden distance learning differs from the traditional one in several features: suddenness, due to an urgent need; lack of necessary high-quality preparedness for it, and well-developed rules; internality, which has grown into a global, worldwide problem due to insufficient social control; popularity; large-scale distribution (penetration into higher education, then into school and preschool education); the imposition of distance learning "from the top-down" without taking into account the democratic aspects of the functioning of society, and the consent of the main participants in the educational process, etc. [2, 3].

Among the main positive characteristics of distance learning, it is advisable to note an individual approach to students, technological efficiency, accessibility, and openness (including that for people with disabilities), as well as mass character and cheapening. Negative characteristics concern technological failures and lack of contact with the teacher, problems of confirming identity during control tests, the complexity of preparing learning materials, and a decrease in the quality of learning [4-7].

Distance learning can contribute to the development of depression. Besides, the lack of live communication and support in the course of studying at the university increases the risks of the students' expulsion. Changes in the learning process lead to anxiety and uncertainty, to the "collective frustration" of teachers [7]. The pandemic destroys the centuries-old culture of pedagogical interaction [2].

In Russia, $60 \%$ of universities successfully coped with the transition to distance learning, which involved more than four million students and about 235 thousand teachers. For teachers, the new form of work was associated with instrumental and methodological problems [8].

The current situation calls into question the competitiveness of traditional universities and indicates the prospects for the development of "hybrid" universities that combine the characteristics of traditional and online universities, as well as for the emergence of individual online universities. The consequences of the pandemic affect the training of practice-oriented specialists and the need to develop innovative areas based on the use of modern technologies. Virtual training offers various conference modifications, virtual cafes, telemedicine, etc. [9-13].

For the administration, distance learning has become a means of implementing their selfinterests. At that, formal indicators of the educational process are becoming the leading ones [6]. The insufficient level of distance learning is observed in many countries $[2,6,14]$, which leads to the need to find ways to resolve the current situation in the context of the pandemic. Besides, the issue of improving the learning efficiency of psychology students in the context of the pandemic has not been sufficiently studied. 


\section{Methods}

The research was conducted in Moscow universities among psychology students (93 students) and university teachers (40 people). To perform the set tasks, the following methods were used: theoretical analysis and generalization of scientific literature, a survey of teachers and students, and the method of qualitative content analysis.

\section{Results}

The survey conducted among the teachers and students identified the following categories of answers: disadvantages of distance learning from the perspective of teachers, and that from the perspective of students, advantages of distance learning from the perspective of teachers, and that from the perspective of students, prospects of distance learning from the perspective of teachers, and that from the perspective of students.

1) Among the disadvantages of distance learning, teachers noted digital incompetence of the educational process participants; an outdated platform for training provided by the university; the difficulty of obtaining feedback; lack of distance learning quality; nonreimbursed material costs of teachers to provide technical support process; lowering of the social status of teacher; increase in conflicts with the students in a situation of chronic stress; daily checks by the university administration.

2) Among the shortcomings, the students named larger volume of homework assignments, insufficient time for preparation; technical difficulties; reduced time for developing the practical skills of psychologists-consultants, and a decrease in the training quality of a practical psychologist.

3) Among the advantages of distance learning, teachers pointed out the provision of security in the context of the pandemic; and the possibility of simultaneous work with foreign and Russian students. For students, the positive aspects were constant access to video lectures and course materials, as well as easier process of passing tests and exams due to a clear scoring system in an electronic environment.

4) The expected negative consequences of the transition to distance learning for teachers were the following: teaching staff reduction; reduced wages and increased workload; replacement of highly qualified teachers' work with automated systems; emotional burnout of teachers; increased risks of closing branches due to the concentration of financial flows in the parent universities. The students noted the inflated cost of educational services (the cost of distance learning was the same as the cost of full-time learning); reduced quality of education; difficulties in improving their professional level as future practical psychologists; lack of psychological practice; noncompliance of graduates with the requirements of employers due to the insufficient level of practical training.

5) The expected positive consequences for both teachers and students were reduced to ensuring health security in the context of the pandemic, providing constant free access to the electronic environment of the university; accumulating experience in working in a remote environment, and improving their technical knowledge and skills.

\section{Discussion}

Analysis of the obtained results has allowed confirming the theoretical phase of the research concerning the lack of the necessary skills in the educational process participants when working with remote technologies; insufficient technical support of distance learning; reduction in the quality of students' training, and the lack of interaction between teachers and students. 
Based on a qualitative analysis of the responses of students and teachers, the authors have formulated the following recommendations:

- moving individual practical training courses and work experience internship for the period of exiting the distance learning mode, while focusing on the disciplines of noncore and theoretical training blocks during the pandemic;

- eliminating the shortcomings of the electronic educational environment of universities;

- freeing teachers' time from formalized, bureaucratic work for the high-quality design of electronic teaching medium, case studies, video lectures, presentations, and other course content;

- opening access to videos of sessions of leading psychological consultants;

- providing access to free online practical conferences related to the activities of practical psychologists, as well as webinars of professional psychological and psychotherapeutic communities;

- conducting remote master classes of psychologists-practitioners of university departments to improve the students' professional training level;

- providing support to teachers and students on the part of qualified representatives of the administration and the technical department, providing support on the days of classes at the university (including Saturday and Sunday);

- providing psychological support for distance learning participants, as well as daily availability of online consultants;

- ensuring reimbursement by the university of financial expenses of teachers for technical support of distance learning, monthly support of educational platforms, such as Zoom, etc.

\section{Conclusion}

Students and teachers must reflect on the experience gained in new conditions to improve their professional training $[15,16]$. It has been revealed that in the context of the pandemic, universities demonstrated a contradictory system of conducting classes in practice-oriented specialties. For psychology students, the distance learning format has significant drawbacks when training the skills of psychological counseling, preparing for psychocorrective and psychodiagnostic activities. Several practical disciplines (such as Psychological counseling, Client-centered psychological counseling, Existential counseling, and psychotherapy, etc.) should be conducted in the full-time format under the supervision of a qualified specialist.

In the context of the pandemic, it is necessary to develop new requirements for the training of practical psychologists, taking into account the improvement of technical means and methods of psychological assistance, which is the subject of authors' further research.

\section{References}

1. M.L. Anderson, S. Turbow, M.A. Willgerodt, G.W. Ruhnke, Society of Hospital Medicine, 15(5), 287-291 (2020). https://doi.org/10.12788/jhm.3431

2. E. Al L. Abdulrahman, F. I. Abdelrahim, M. A. Fathi, H. A. A. Rafdan, Technology in Society, 63, 101317 (2020). https://doi.org/10.1016/j.techsoc.2020.101317.

3. M.B. Pitt, S.-T. T. Li, M. Klein, Academic Pediatrics, 20(6), 733-734 (2020). https://doi.org/10.1016/j.acap.2020.06.002

4. N. Bukeikhanov, S. Gvozdkova, E. Butrimova, Russian Regions: Looking Into the Future, 7(2), 62-75 (2020)

5. A.S. Gotlib, Bulletin of Samara State University, 1(123), 15-22 (2015) 
6. A.D. Ivanova, O.V. Murugova, Open Education, 24(2), 4-16 (2020)

7. D.V. Lvov, Professional Education in the Modern World, 10(1), 3456-3464 (2020)

8. D.A. Shtykhno, L.V. Konstantinova, N.N. Gagiev, Open Education, 24(5), 73-81 (2020)

9. R. Blankenburg, P. Poitevien, J. Gonzalez del Rey, L. Degnon, Academic Pediatrics, 20, 757-759 (2020). https://doi.org/10.1016/j.acap.2020.05.025

10. T.M. Makhamatov, T.T. Makhamatov, S.T. Makhamatova, Lecture Notes in Networks and Systems, 129, 816-825 (2020). https://doi.org/10.1007/978-3-030-47945-9_87

11. B. Rangiwai, B. Simati-Kumar, Te Kaharoa, 15(1), 1-14 (2020). https://doi.org/10.24135/tekaharoa.v15i1.290

12. A. Sufian, A. Ghosh, A.S. Sadiqb, F. Smarandache, Journal of Systems Architecture, 108, 1-30 (2020). https://doi.org/10.1016/j.sysarc.2020.101830

13. C.M. Toquero, Pedagogical Research, 5(4), em0063 (2020). https://doi.org/10.29333/pr/7947

14. A.M. Zha, L.S. Chung, Sh.S. Song, J.J. Majersik, A.L. Jagolino-Cole, Neurology, 95, 404-407 (2020). https://doi.org/10.1212/WNL.0000000000010029

15. O.I. Kayasheva, Z.G. Khanova, EurAsian Journal of BioSciences, 13, 2051-2056 (2019)

16. O.I. Kayasheva, V. Kislyakov, International Journal of Psychosocial Rehabilitation, 24(08), 13978-13989 (2020). https://doi.org/10.37200/IJPR/V24I8/PR2801378 